\title{
A computer program for on-line measurement, storage, analysis and retrieval of urodynamic data
}

\author{
R. van Mastrigt \\ Dept. of Urology, Erasmus University Rotterdam, P.O. Box 1738, 3000 DR Rotterdam, The Netherlands
}

\begin{abstract}
A computer program is presented which allows for direct connection of a minicomputer to a urodynamic set-up. The program stores measured pressure and flow data in a random access disc file with minimal intervention of the urodynamicist, and enables the direct application of a number of methods of analysis to the data. The program is modular, and other analysis methods are easily added. Results of analyses are stored in the same disc file, and both results and measured data can be quickly and easily retrieved. The program is written in FORTRAN; hardware-dependent functions (analog input, graphics display, and random access disc storage) are implemented in subroutines (partly assembler) which can easily be replaced.
\end{abstract}

Urodynamics Urinary bladder Force-velocity relation Hill model Pressure-flow analysis Contraction velocity FORTRAN program

\section{INTRODUCTION}

Urodynamics is concerned with the application of physical models, and the making and interpretation of measurements of physical quantities such as pressure, volume and flow, in urology. In most urodynamic measurements at least the pressure in the urinary bladder and the rate of urine flow during micturition are measured.For the analysis of these data complicated models are often used, which make use of a computer necessary. A computer program for urodynamics might be of any of the following three types:

(1) A dedicated database management system, which enables storage and retrieval of parameters from a keyboard [1].

(2) A program for a dedicated analysis of data recordings, on magnetic tape for instance [2].

(3) A program which combines the functions (1) and (2) above with direct on-line measurement of the data.
A program of the latter type has been developed. It does not pretend to cover all urodynamic activities, but is a modular system which takes pressure and flow data direct from the urodynamic experiment in a way which demands minimal attention from the urodynamicist, stores them in a random access disc file, and offers a choice of analyses which can be applied directly to the data. At present the following analyses are implemented:

(1) Calculation and display of a force-velocity relation from isometric contractions, according to Hill's model [3] using a preset, assumed series elasticity $[4,5]$. Either spontaneous contractions observed during the filling of the bladder, or the pressure rise observed just before micturition starts, may be analysed. The relation is fitted with a hyperbolic Hill equation with preset curvature [6].

(2) Display of a complete micturition in the form of a pressure-flow relation, taking into account the delay between the moment at which the 
urinary stream leaves the bladder and the moment at which it is recorded by the flow meter. Calculation and display of a pressure-contraction velocity relation taking into account the residual urine in the bladder after micturition. This relation too is fitted with a hyperbolic equation, with manual intervention [7].

Results of both types of analysis are stored directly in the same random access disc file as the measured data. New types of analyses can easily be added. A number of separate utilities are compatible with the disc structure employed and are used for this data:

(1) A program for interactive changing of data and parameter values in the random access disc file (used mostly to correct errors).

(2) A program for batch listing of the resulting parameters.

(3) A program for performing simple standard operations on specified parameters in batch.

(4) A program for batch plotting of the relations calculated from complete micturitions (analysis (2) above).

(5) A program for batch analysis of isometric contractions in terms of phase plots [8].

\section{HARDWARE AND SOFTWARE REQUIRE- MENTS}

The program is written in FORTRAN and currently runs on a Texas Instruments $980 \mathrm{~B}$ minicomputer with 48 Kwords of main memory and two Diablo 2.5 Mbyte removable disc cartridge drives. The program is overlayed in three levels and runs in about $12 \mathrm{Kwords}$ of memory. It would occupy about $30 \mathrm{Kwords}$ in a non-overlayed version. In the normal situation the program functions with a remote $A / D$ converter and graphics terminal, situated near the urodynamic set-up, and connected to the computer via a telephone line (212 type modems, 1200 baud) [9]. This means

that, as soon as the $\mathrm{A} / \mathrm{D}$ converter starts sending data via the line, the terminal is dead. The $A / D$ converter samples 2 channels, bladder pressure and urine flow rate, at 10 samples/second. The graphics terminal is an ADM3 with Retrographics; i.e., Tektronix 4010 compatible. The following functions are hardware dependent and are implemented in subroutines which must be rewritten when the program is transported to another system.

\subsection{Analog input}

The subroutine AD2NOS reads one complete sample coming in at the RS232 port connected to the modem. As well as the sampled analog input channels, information on the setting of four sense switches on the A/D converter system is obtained. These sense switches form the only means of communication between the urodynamicist and the program during measuring. A subroutine IKAR2 is used for sending out ASCII control characters in

\section{TABLE 1}

Record types in the disc file

\begin{tabular}{lll}
\hline Key & Record & \\
\hline NRSS & 4 integers denoting: last contraction stored \\
& $\begin{array}{l}\text { last contraction pro- } \\
\text { cessed } \\
\text { last flow stored } \\
\text { last flow processed }\end{array}$
\end{tabular}

ABEL

PA 'number' 20 words results of contraction measurements from contraction 'number'

PD 'number' 50 words measured pressure data from contraction 'number' followed by 4 sequential records of 50 words each

FA 'number' 20 words results of flow measurements, from flow 'number'

FD 'number' 50 words measured flow data, from flow 'number', followed by $N-1$ sequential records of 50 words each. $N$ is the first word of record FA 'number'

FP 'number' Same structure as FD 'number' but contains measured pressure during flow 
order to start and stop A/D conversion, etc. A subroutine CRTAIN creates a separate program task which buffers incoming data. This task is finished by the subroutine KILTAS. In single user systems these last two subroutines are probably not necessary.

\subsection{Disc storage}

The subroutine KEYWR is used to store and retrieve keyed and unkeyed records in the one disc file employed. One single key consisting of two characters and an integer or a second pair of characters is used. The record types used are shown in table 1. The subroutine REREC reads one keyed record and a specified number of the following sequential records using KEYWR.

\subsection{Graphics output}

For generating graphic displays on the 4010 compatible terminal a set of subroutines is used which are compatible with conventional pen plotten routines (table 2 ).

\subsection{Various subroutines}

The subroutines OPEN and CLOSE open and close devices, respectively. IDATE obtains the date from the operating system. The subroutine RE$\mathrm{ACH}$ prompts for keyboard input and checks. It uses a FORTRAN extension.

\section{TABLE 2}

Pen plotter compatible subroutines used for graphic output on the 4010 compatible terminal

\begin{tabular}{|c|c|}
\hline Subroutine & Function \\
\hline VPLOTS & Initializes \\
\hline VPLOT & Plots points and draws vectors \\
\hline VOFFSET & $\begin{array}{l}\text { Sets coordinate offets and scaling } \\
\text { factors }\end{array}$ \\
\hline VLINE & Plots arrays into line graphs \\
\hline VAXIS & $\begin{array}{l}\text { Draws } X \text { and } Y \text { axes including tick } \\
\text { marks, scale annotations and axis labels }\end{array}$ \\
\hline VLABEL & $\begin{array}{l}\text { Labels a graph in upper right } \\
\text { corner }\end{array}$ \\
\hline VCURSOR & $\begin{array}{l}\text { Moves the cursor around, using } \\
\text { keyboard keys }\end{array}$ \\
\hline
\end{tabular}

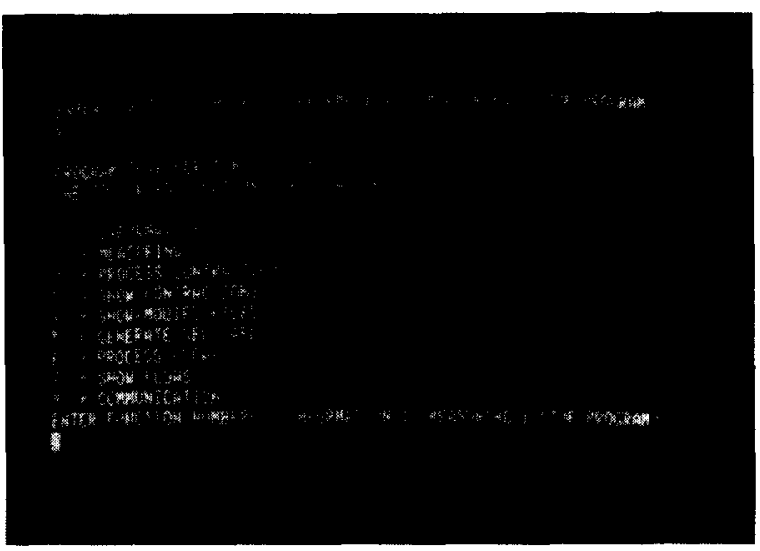

Fig. 1. Display showing prompt line and information displayed upon entering function 0 .

\section{DESCRIPTION OF PROGRAM}

\subsection{Prompt line and available functions}

The program has been split up into a number of functions. Upon starting, a prompt line requests what function is to be performed. Upon leaving a function the prompt line is repeated. The subroutine $\mathrm{REACH}$, which displays the prompt line, repeats it on any input other than a legal function number followed by a decimal point and return. Therefore, if (modem) connections are made when the program is already running, and the prompt line has already been emitted, it can be evoked by pressing return or any other illegal input. Function number 0 . displays a list of available functions. See Fig. 1 for prompt line and output of function 0 .

\subsection{Function 1: measuring}

Figure 2 shows a flowchart of function 1, measuring. At entry a two character patient ID is requested, if $\$ \$$ is entered the function is left. Otherwise A/D conversion is started, and the pressure signal is recorded in a 'circular' file that is 250 samples (i.e., 25 seconds) long. Upon pressing sense switch 4 this file (i.e., the last 25 seconds of pressure data) is stored on disc using a contraction number identifier which is automatically increased. Sense switch 3 makes the system flow 


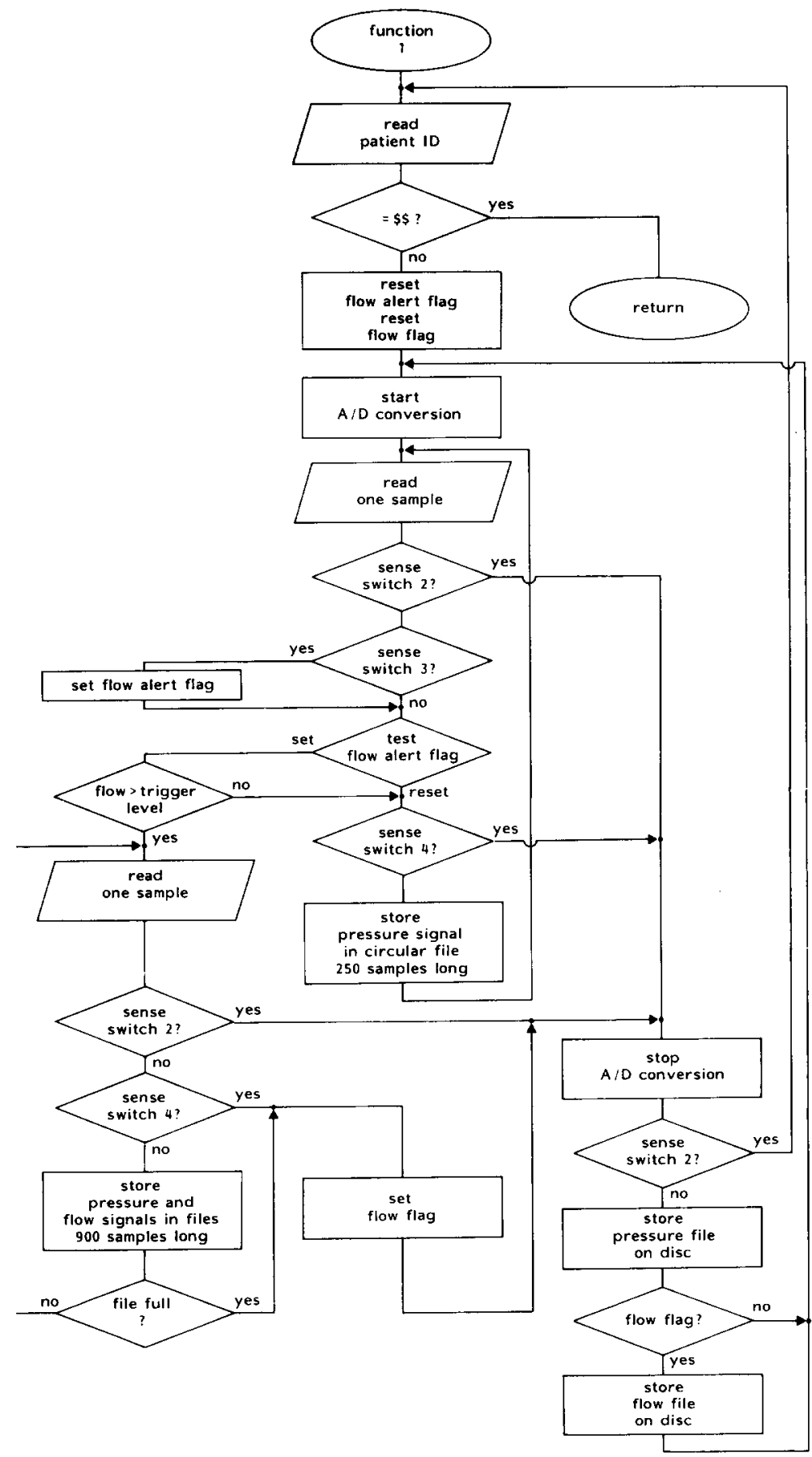

Fig. 2. Flowchart of function 1; measuring. 


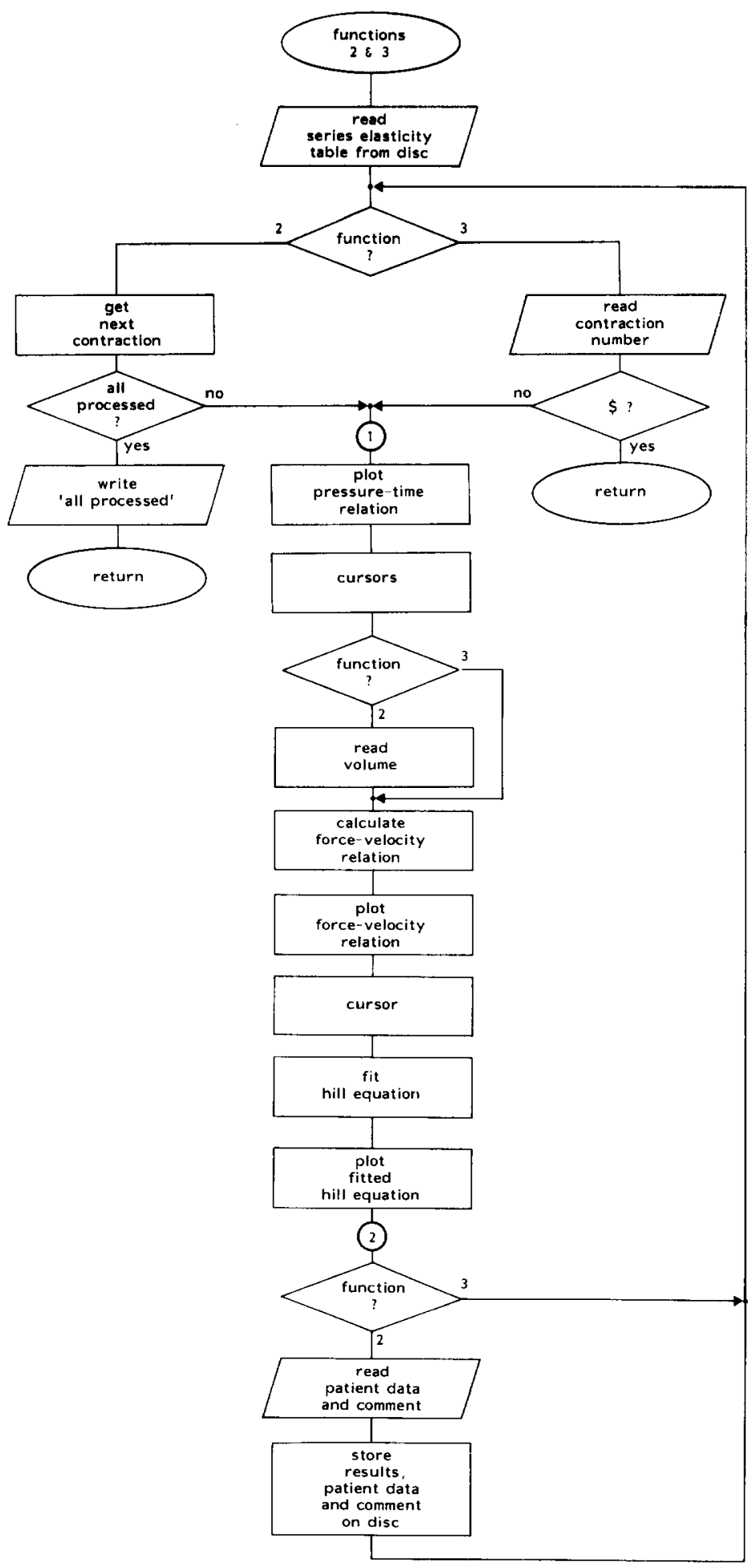

Fig. 3. Flowchart of functions 2 and 3; process and show contractions. 


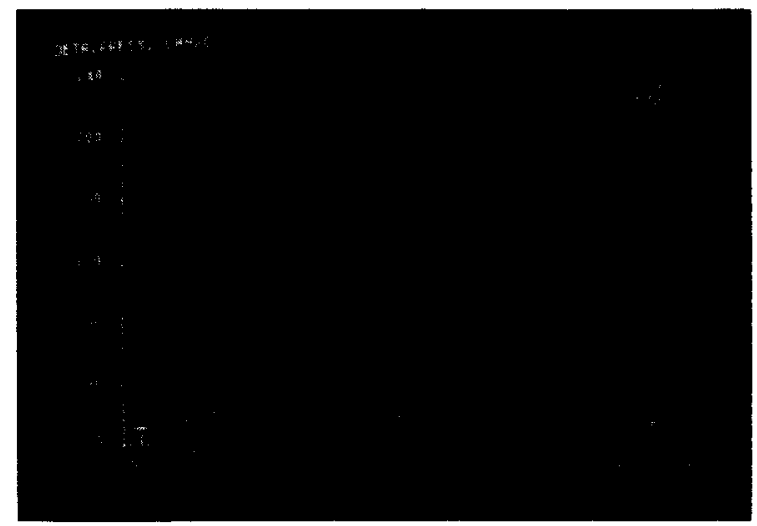

Fig. 4. Graphic display of measured pressure during a contraction as a function of time. Generated in functions 2 and 3.

alert. If the flow rate exceeds a trigger level, samples are read into two separate files, one for flow rate and one for pressure, 900 samples long. Either the completion of these files, or the depressing of sense switch 4, initiates storage on disc of both flow rate and pressure during flow, using an automatically increased flow identifier. In addition the contraction immediately preceding the onset of flow, which is present in the circular 250 sample file, is stored on disc with its own contraction identifier. In all cases sense switch 2 returns control to entering the patient ID.

\subsection{Functions 2 and 3: process and show contrac- tions}

Functions 2 and 3 are almost identical. A flowchart of these functions is shown in Fig. 3.

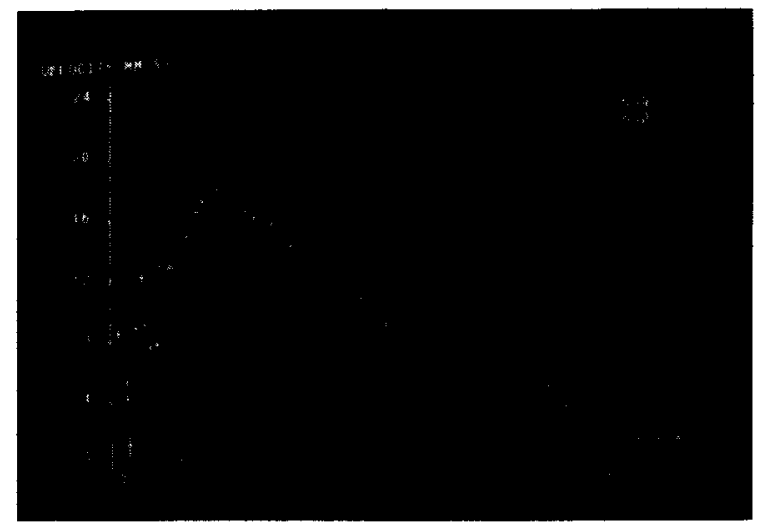

Fig. 5. Graphic display of force-velocity relation calculated from the data shown in Fig. 4. Generated in functions 2 and 3.

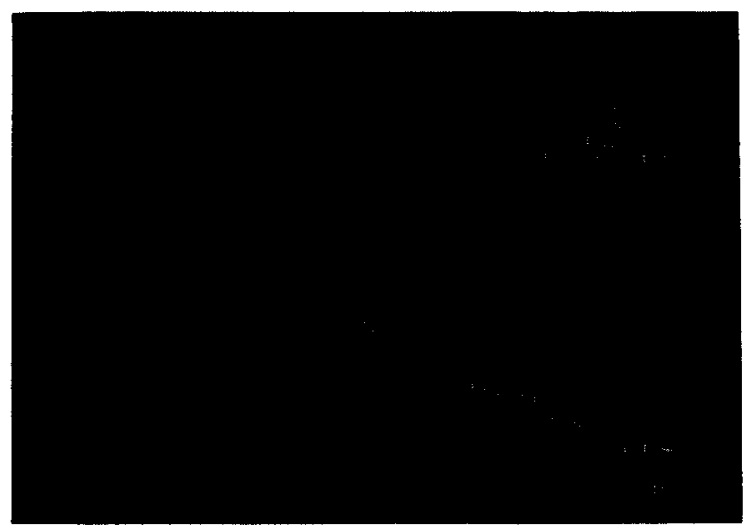

Fig. 6. Graphic display showing hyperbolic Hill curve fitted to the data of Fig. 5. Generated in functions 2 and 3.

Every measured contraction has to be processed (function 2), and at this time extra patient data are entered, which are stored on disc together with the results of the calculations. This process is performed sequentially on all measured contractions using a counter in the disc record with key NRSS. Following processing, contractions and the associated calculations can be 'shown' as often as required, in random order. Both 'process' and 'show' contractions involve a plot of the measured pressure as a function of time on the graphics terminal (see Fig. 4), the setting of cursors to mark the beginning and end of a part of the curve which is of interest, and the calculation of a force-velocity relation from the contraction using Hill's model [3] with an assumed series elasticity characteristic read from a disc table. The force-velocity relation is displayed on the terminal (see Fig. 5), a region of interest is again indicated using a cursor, and a hyperbolic Hill equation is fitted to the indicated data and plotted on the screen (see Fig. 6).

\subsection{Function 4: show and modify files}

Function 4 offers a limited possibility of entering or reading a keyed or unkeyed record in the disc file. The function is not very easy to use and is merely intended for emergency situations.

\subsection{Function 5: generate series elasticity table}

Function 5 generates a series elasticity table in the disc file and has to be run once every time a 
new disc file is used. The generated elastic characteristic is of the form:

$F=G \times L \exp (b \times L)$

where:

$F=$ force exerted on the series elasticity;

$L \quad=$ its length;

$b$ and $G=$ parameters.

The parameters $b$ and $G$ have to be entered.

\subsection{Functions 6 and 7: process and show flows}

Functions 6 and 7 are identical to functions 2 and 3 with respect to the 'process' and 'show' differences. Apart from using other pointers the

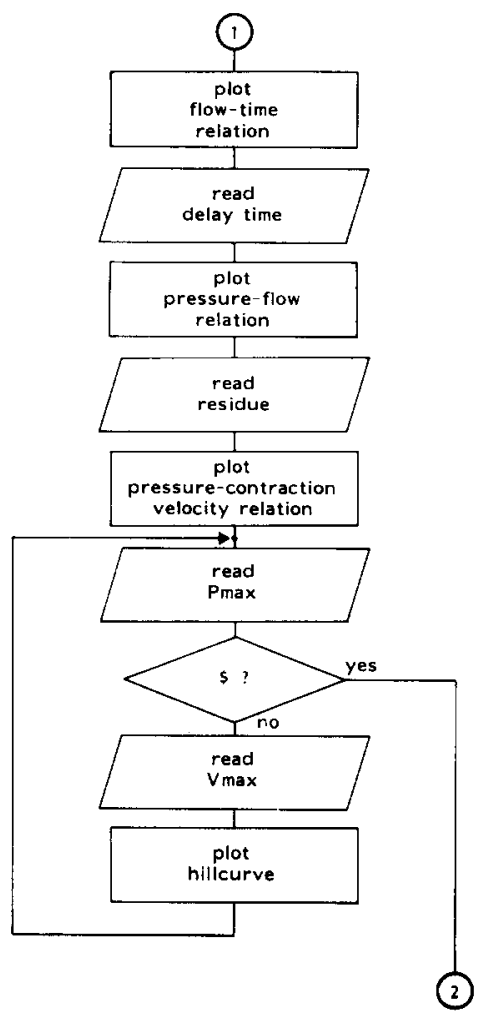

Fig. 7. Flowchart of part replacing the part between terminals 1 and 2 in Fig. 3 to yield flowchart of functions 6 and 7; process and show flows.

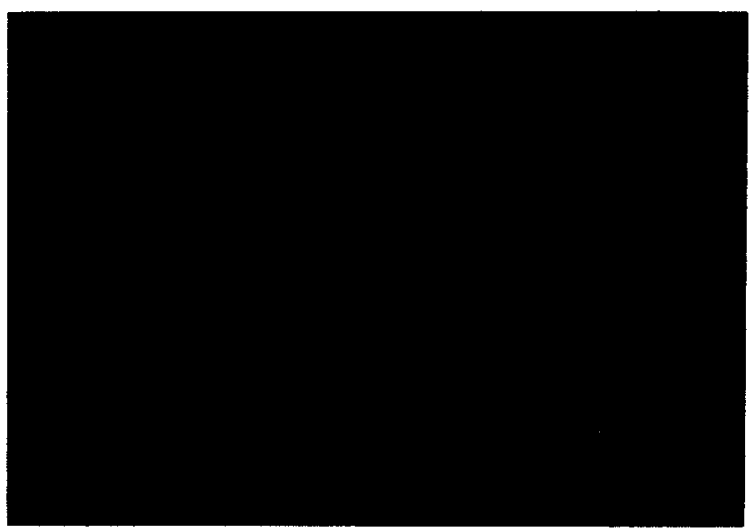

Fig. 8. Graphic display of measured urinary flowrate as a function of time. Generated in functions 6 and 7.

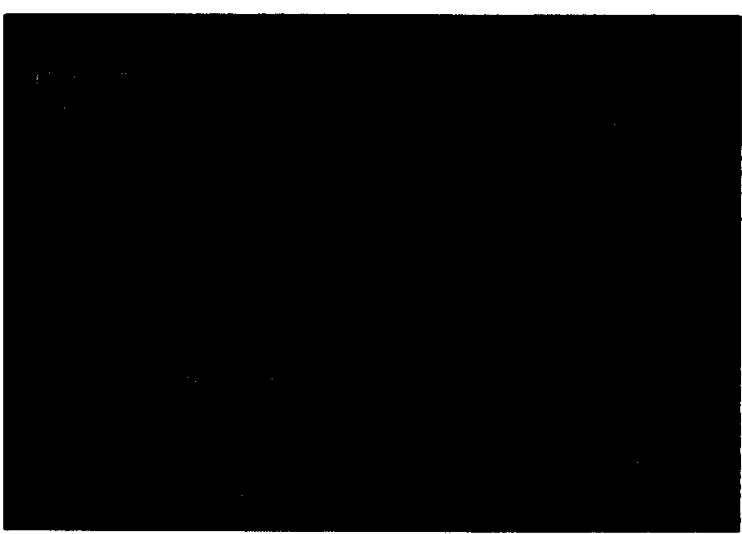

Fig. 9. Graphic display of measured urine flow rate as a function of pressure in the urinary bladder. Corrected for delay in measurement of flow rate, using a delay of 1.0 seconds in this case. Same data as displayed in Fig. 8. Generated in functions 6 and 7 .

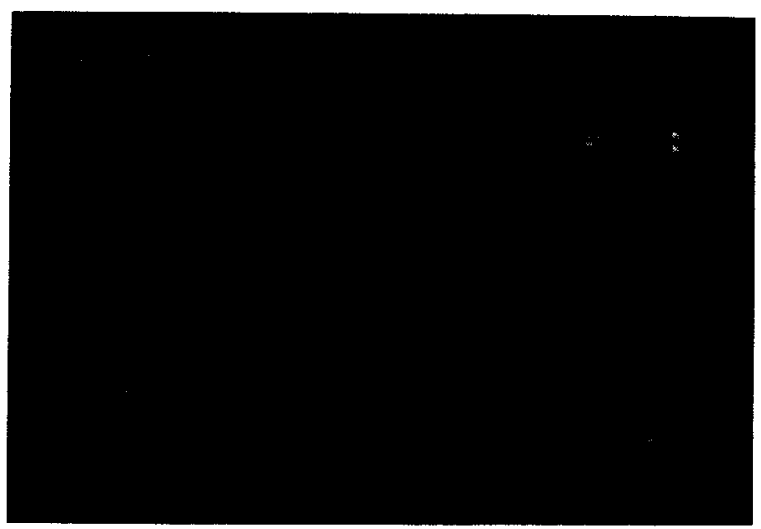

Fig. 10. Graphic display of calculated urinary bladder contraction velocity as a function of bladder pressure. Calculated from the data shown in Fig. 9 in functions 6 and 7. 


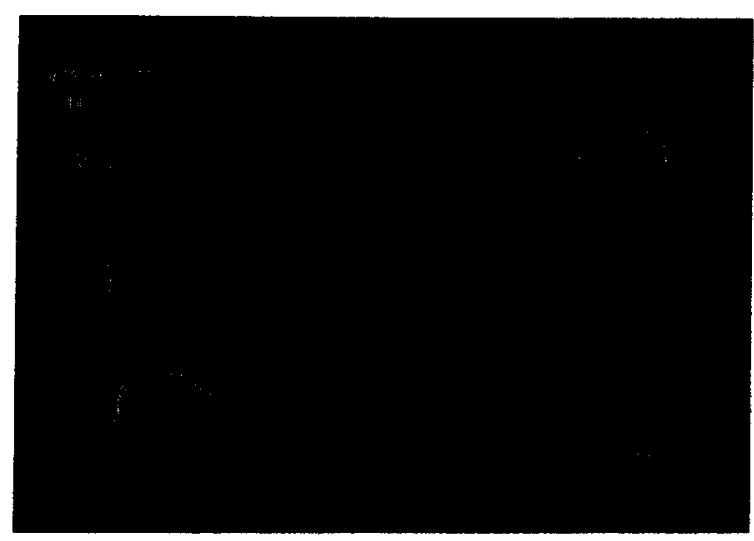

Fig. 11. Same display as Fig. 10 with a manually fitted hyperbolic curve. Generated in functions 6 and 7 .

part between terminals 1 and 2 of the flowchart shown in Fig. 3 can be replaced by Fig. 7 to give the appropriate flowchart. Following a plot of the measured flow as a function of time (see Fig. 8), the delay time between pressure and flow is requested. In case of 'show' the value entered during 'process' can be used by entering a specific symbol. Next a pressure-flow relation is plotted as shown in Fig. 9, and the residual urine which was left in the bladder after micturition is requested (same option as above). The final plot, of pressure as a function of contraction velocity, is shown in Fig. 10. By entering the intersections on the pressure and velocity axes, a hyperbolic curve can be drawn several times in succession (see Fig. 11 for an example), until a satisfactory approximation is obtained. The parameters of the last curve to be drawn are stored on disc together with the requested patient data.

\subsection{Function 9: communication}

(Function 8 has been deleted.)

Function 9 offers the possibility of entering, on the (remote) terminal next to the urodynamic setup, a message which is displayed at the logging terminal of the computer.

\section{CONCLUSIONS}

The program described above has been in use for almost two years so that the most frequently occurring bugs have been removed.At the moment it runs typically for longer than 10 days before a problem occurs. The largest source of errors is the modem connection, which tends occasionally to start or stop A/D conversion spontaneously. For transport of the program to another system, the subroutines listed in table 2 would have to be rewritten. In the near future the program will be transferred to a DEC PW350 personal computer. The new version of the program will run also on any PDP11 RSX system. The addition of further methods of analysis to the program is fairly easy. It involves writing a new function module, and might involve the addition of disc records with different keys for storing results. The addition of other measurements might however be more complicated. It would be possible, for instance, to store ALL measured pressure data at a lower sampling rate (i.e., to select one out of every so many samples) in order to have a complete cystometry curve. Due to lack of suitable analysis techniques this has not (yet) been realized. In such a case function 1 , measuring would have to be changed drastically.

\section{REFERENCES}

[1] J.R. Woodside and F.M. Morris, A computer program for the storage and retrieval of urodynamic data, Neurourol. Urodynam. 1 (1982) 313-318.

[2] R. van Mastrigt, Constant step approximation of multi-exponential signals using a least squares criterion, Comput. Biol. Med. 7 (1977) 231-247.

[3] A.V. Hill, The heat of shortening and the dynamic constants of muscle, Proc. Roy. Soc. B 126 (1938) 136-195.

[4] R. van Mastrigt and D.J. Griffiths, The contractility of the urinary bladder, Urol. Int. 34 (1979) 410-420.

[5] R. van Mastrigt, Determination of the contractility of children's bladders from isometric contractions. Urol. Int. 38 (1983) 354-362.

[6] R. van Mastrigt, Fitting the Hill equation to experimental data, IEEE Trans. Biomed. Eng. 27 (1980) 413-416.

[7] D.J. Griffiths and R. van Mastrigt, The routine assessment of detrusor contraction strength, Neurourol. Urodynam. (1984) in press. 
[8] R. van Mastrigt and J.J. Glerum, Electrical stimulation of smooth muscle strips from the urinary bladder of the pig (1984) submitted.

[9] R. van Mastrigt, H.R. Thon and H. van de Giessen, Analog data collection via a telephone line: A micro- processor-based remote-controlled data-acquisition system, Med. Biol. Eng. Comput. 21 (1983) 229-231.

[10]. R. van Mastrigt and J.J. Glerum, In vitro comparison of isometric and stop-test contractility parameters for the urinary bladder (1984) submitted. 